\title{
Monitoring of the nano-structure response of natural clay under mechanical perturbation using small angle X-ray scattering and digital image correlation
}

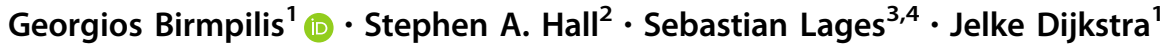

Received: 10 July 2018 / Accepted: 2 June 2019/Published online: 19 June 2019

(c) The Author(s) 2019

\begin{abstract}
This paper introduces a new experimental method to monitor the evolving intra-particle, nanometre-scale response during hydro-mechanical tests on undisturbed wet clay samples using small angle X-ray scattering (SAXS). The method uses a newly developed miniature plane-strain one-dimensional compression cell that facilitates simultaneous full-field surface displacement measurements using digital image correlation and SAXS measurements. The 60-120 s acquisition times offered by SAXS at synchrotron facilities are beneficial to study time-dependent mechanisms in clays. The experimental results presented indicate that, for the natural sensitive clay tested in this work, no significant structural changes occur at the intra-particle scale during loading, even when large strains are measured at the macro-scale. In addition, changes that are observed at this scale occur after the end of perturbation, i.e. the creation of new intra-particle structures. Further information is obtained on the orientation evolution of the fabric by the comparative analysis of the individual mineral component response.
\end{abstract}

Keywords Clay $\cdot$ DIC $\cdot$ Directional anisotropy $\cdot$ Nanoscale $\cdot$ Real-time $\cdot$ SAXS

Georgios Birmpilis

georgios.birmpilis@chalmers.se

Stephen A. Hall

stephen.hall@solid.lth.se

Sebastian Lages

sebastian.lages@fau.de

Jelke Dijkstra

jelke.dijkstra@chalmers.se

1 Division of Geology and Geotechnics, Department of Architecture and Civil Engineering, Chalmers University of Technology, 41296 Gothenburg, Sweden

2 Division of Solid Mechanics, Lund University, 22100 Lund, Sweden

3 Max IV Laboratory, Lund University, P.O. Box 118, 22100 Lund, Sweden

4 Institute for Crystallography and Structural Physics (ICSP), Friedrich-Alexander-Universität Erlangen-Nürnberg, 91058 Erlangen, Germany

\section{Introduction}

The emerging hydro-mechanical response observed at continuum scale in fine-grained soils results from a combination of electrical, capillary and frictional forces in addition to cementation (bonding) between the soil particles [28]. In particular, the evolution of inter-particle bonds in clays leads to the distinctive emerging mechanical behaviour of the colloidal fraction of soil and is responsible for the thixotropic mechanisms in sensitive clays, i.e. a reversal of micro-structure to a thermodynamically stable state after mechanical perturbation [1]. In geomechanics, this is associated with a time-dependent increase in stiffness and strength due to the recovery of bonds [24]. In a three-phase material (soil, water, air), inter-particle bonds are the result of complex surface chemistry. Interparticle and intra-particle forces are principally controlled by the presence and chemical composition of water, which can be bonded to clay particles as well as free in the pore space. The product of these forces in equilibrium is the spatial arrangement of particles, responsible for the macroscopic hydro-mechanical performance of clay soils. 
Several theoretical conceptual models regarding fabric and structure of clays have been postulated. Here, the term soil fabric refers to the geometrical arrangement of the particles and the term soil structure is used for the combination of fabric and additional inter-particle bonding. The latter depends on physical and chemical conditions $[8,21]$. It should be noted that particle mineralogy (and their associated physico-chemical response), in addition to particle arrangement, has a significant role in the response of the clay colloidal system. For instance, the mineral composition of sensitive (quick) clays consists typically of a dominant stable mineral (illite) and an expandable one (montmorillonite) as a smaller fraction [31].

Two of the first and dominant conceptual models on clay micro-structure are the card house [25] and the honeycomb structure [5], which describe the structure that results from gel formation and focus on the type of contact between clay particles. More realistic models for the clay response have subsequently been formulated, though they still are mainly based on experimental observations of clay suspensions [6]. The latest generation of models approaches the complete physico-chemical response between the clay minerals and the surrounding water using Molecular Dynamics (MD) simulations, most notably Ebrahimi et al. [12] and Brochard et al. [4]. These advanced methods, whilst very promising, still would benefit from more experimental evidence on the modelled length scale $(n m-\mu m)$. Given that the experimental measurement of forces on the microstructural scale remains unresolved, the experimental study is principally focused on the resulting evolution of fabric.

The majority of theoretical models used in geomechanics have been primarily based on investigations using invasive experimental methods, such as Scanning Electron Microscopy (SEM), Mercury Intrusion Porosimetry (MIP) or both $[2,11,15-17,30,32]$. These methods require sample preparation that drains or freezes the pore water, or demands the intrusion of other replacing fluids at high pressures and potentially affects the material structure and properties. Recently, Cryo-SEM and Cryo-WAXS, methods that use superior freezing techniques, have been used to examine the effect of such invasive techniques on the natural clay micro-structure and porosity. These studies concluded that traditional sample preparation techniques (e.g. plunge freezing) do affect the clay structure significantly $[9,10]$. Furthermore, the study of the evolution of the micro-structural properties of clays is complicated by the need to take samples at several stages in the process, which requires a series of tests terminated at different stages for subsequent sample collection.

Given the above discussed limitations of existing experimental methods to investigate clay structure, there is a clear need for non-invasive techniques that enable information to be gathered on the evolving inter-particle and intra-particle systems.

In this paper, the results are presented from a new experimental procedure based on Small Angle X-ray Scattering (SAXS) for which no additional sample preparation is required. The evolution of particle orientation during loading, which can be determined using SAXS, is an indirect way to observe global fabric changes. Hence, for the first time the clay inter-particle and sub-particle structure evolution in unaltered wet clay samples can be studied. Furthermore, the non-destructive nature allows the link between structure and emerging hydro-mechanical soil behaviour to be studied during mechanical loading.

\section{Experimental methodology}

The experiments were designed to test a clay in its natural, undisturbed, wet state in a 1D compression test whilst performing both Small Angle X-ray Scattering (SAXS), to monitor structural changes at the intra-particle scale, and full-field surface displacement measurements over the sample area via Digital Image Correlation (DIC). It is of utmost importance to obtain the SAXS and DIC image data at a sufficiently high acquisition rate during the mechanical loading without the need for test interruptions or subsequent destructive post-mortem analyses.

\subsection{Cell for mechanical loading}

1D compression loading is selected since the macroscopic response is uniform and therefore can easily be correlated with the bulk measurements from SAXS, which provides particle-level observations. As such, the test cell was designed to accommodate simultaneous SAXS, which requires transmission of X-rays, and DIC measurements that require optically transparent cell walls and an adequate speckle pattern on the surface of the sample.

It is essential to minimise the disturbance of the specimens during sampling from a larger block to retain as much as possible their original structure. Therefore, the test cell is used to sample the specimens from a naturally consolidated clay sample and to confine them during subsequent loading.

Borosilicate glass rectangular capillary tubes from Hilgenberg $\mathrm{GmbH}$ (Article no. 1408964), $6.50 \mathrm{~mm} \times 1.40$ $\mathrm{mm}$ outer dimensions, $0.16 \mathrm{~mm}$ wall thickness, are used (Fig. 1). The requirement for sufficient transmission of X-rays for SAXS through both the confining capillary and the specimen limits the specimen thickness to approximately $1 \mathrm{~mm}$ using the described borosilicate glass capillary tube. The height of the specimen is $29.7 \mathrm{~mm}$. The geometry of the tube ensures that the vertical penetration of 

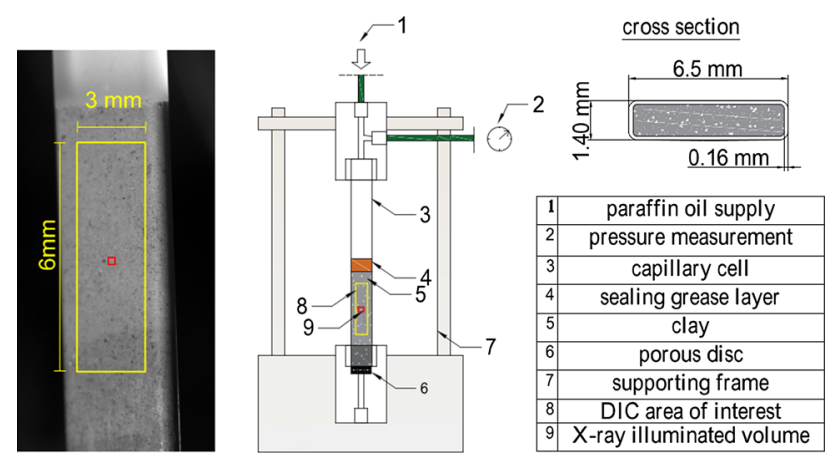

Fig. 1 Summary of the specimen configuration: the capillary 1D compression cell (middle) contains the clay specimen, whilst allows for surface DIC measurements (left) and X-ray transmission through it (cross section on the right)

the tube into the soil during sampling does not strain the total volume of the soil, apart from the zones next to the wall boundaries. Furthermore, a hydrophobic coating is applied to the inner surfaces of the tube to further reduce wall friction during sampling, as well as decreasing the residual adhesion between the sample and the capillary walls.

After the sampling, the capillary forms a confinement cell (Fig. 1). A boundary load is imposed on the top of the sample using liquid paraffin, which does not penetrate the fine-grained sample. An extra layer of semisolid lubricant seals the top of the sample from oil intrusion, as well as protects the specimen from drying in the period between sampling and testing. Pressure measurements are made at the top end and the bottom end forms a free drainage boundary. Pressure is applied using a high precision neMESYS Mid Pressure Syringe Pump (Cetoni GmbH), and the mechanical response of the specimen is recorded simultaneously with SAXS and DIC measurements.

\subsection{Digital Image Correlation}

Surface DIC measurements can be used as an effective macro- and micro-investigation tool in clayey materials as it has been demonstrated by Bornert et al. [3] and Yang et al. [33] for the case of argillaceous rocks. Digital images of the sample surface were acquired using two Prosilica GT6600 29 Megapixel digital cameras (Allied Vision Technologies) in a stereoscopic configuration mounted inside the SAXS hutch. The achieved pixel size was $10 \mu \mathrm{m}$. A TTL signal from the SAXS detector was used to trigger the DIC system, so that every time a SAXS image was recorded DIC images were also taken, similar to Engqvist et al. [13]. Furthermore, the logfile of the SAXS measurement registered the start and the end of the acquisition in laboratory time. The closest reading for the mechanical stress at the final acquisition time is used to plot the stress- strain curve. The uncertainty between the timelines of the both processes (stress control and imaging) stems from the accuracy to which the file creation is known $( \pm 1 \mathrm{~s})$. When compared to the acquisition rate of the image data (60-120 s), this uncertainty is acceptable. In Fig. 2, a schematic diagram of the experimental configuration inside the SAXS hutch is presented.

Vic-3D (Correlated Solutions Inc.) was used for the calibration and processing of the digital images to yield the surface displacement fields and, hence, strain fields corrected for the out-of-plane movements. The stereo calibration was achieved by the use of reference grid images in different positions and rotations as described by the manufacturer [7]. The natural speckle of the clay provided by the granular fraction in the clay (ca. $>60 \%$ coarse particles by mass) offers sufficient contrast for the DIC processing. The latter is judged from the variation in intensity of a selected search window size. The histogram of grey values of the down-sampled 8-bit image is presented in Fig. 3.

\subsection{Small angle X-ray scattering}

SAXS is a non-destructive method, based on the elastic scattering of X-rays that provides bulk measurements of the structure of scatterers in the $\AA$ to $\mathrm{nm}$ range. Very low scattering angles $2 \theta \quad 0.1^{\circ}-10^{\circ}$ allow characterisation of structures in the $\mathrm{nm}$ range desired for this work. Scattering techniques provide reciprocal space-averaged observations over macroscopic volumes. Moreover, scattering techniques have various advantages compared to traditional direct space microscopy for in-situ and time-resolved studies [27], due to their non-destructive nature and the high acquisition rate of measurements. SAXS has already been used for the in-situ study of clay suspensions of smectites [23, 26, 34], but due to the high penetration power of X-rays it can also be applicable to thin natural clay samples of higher density.

SAXS instrument accessibility is continuously increasing by the growth of a large network of conventional laboratory system solutions. In addition, high brilliance sources (synchrotron beamlines) keep improving the acquisition rates for the study of time-dependent

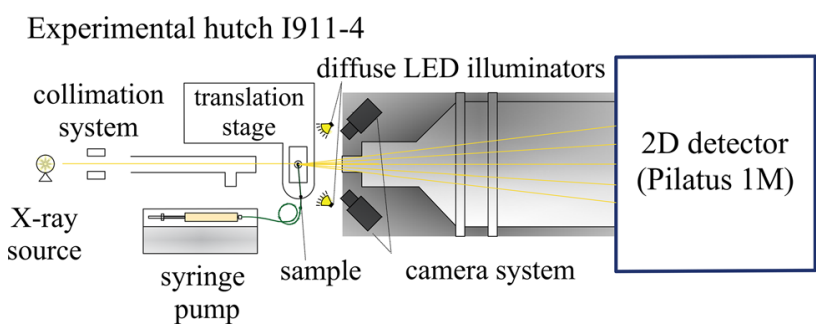

Fig. 2 Schematic diagram of experimental configuration in experimental hutch I911-4 

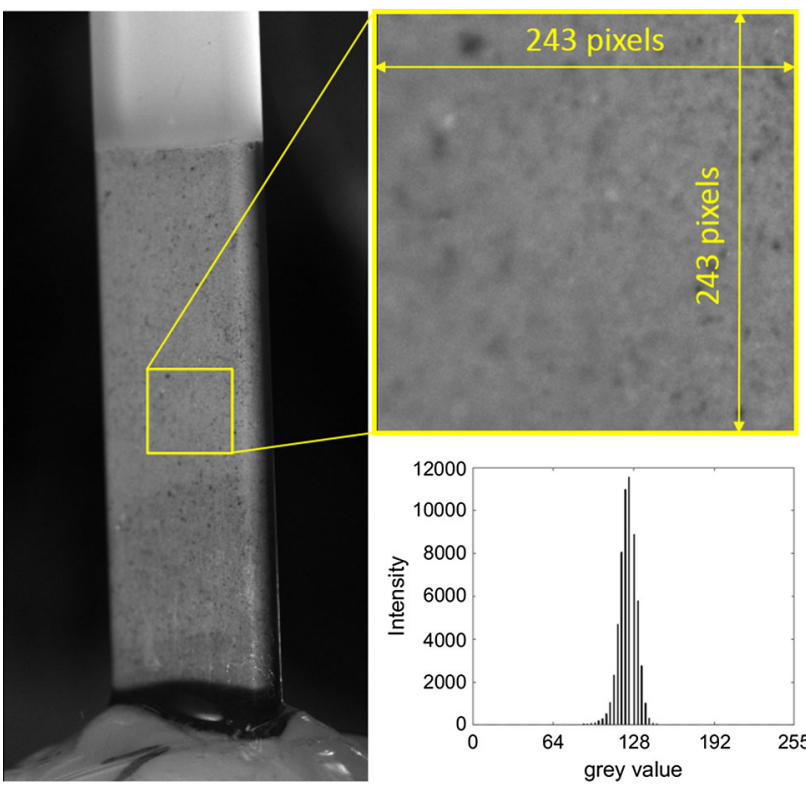

Fig. 3 Silt grains act as the natural speckle which is necessary for the image correlation. An histogram of a characteristic subset is provided

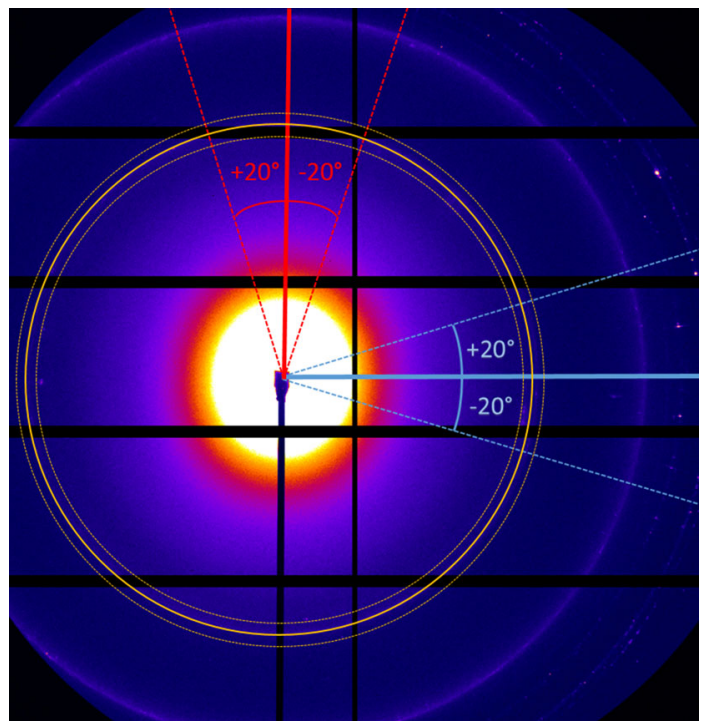

Fig. 4 SAXS 2D normalised intensity matrix. Data are extracted by angular sectors (blue and red) as well as polar plots of intensity through azimuth (yellow ring) averaged for a radial range of 10 pixels (colour figure online)

phenomena, offering new potential to material and engineering sciences. The tests presented in this paper are performed at the I911-4 SAXS beamline at the MAXIV Laboratory in Lund, Sweden [20]. The transmitted X-ray beam wavelength is $\lambda=0.91 \AA$ with a spot size incident on the sample of $0.3 \times 0.3 \mathrm{~mm}^{2}$. The distance to the detector is $0.96673 \mathrm{~m}$, resulting in a $q$-range of $0.01-0.75 \AA^{-1}$. The temperature in the experimental hutch was relatively constant at $25{ }^{\circ} \mathrm{C}$ during the tests.
X-ray scattering-based techniques transform electron density heterogeneities of matter in real space to a reciprocal $q$-space according to Bragg's law (Eq. 1) [14]:

$n \lambda=2 d \sin \theta$

where $d$ is the distance between layers of scatterers, $\lambda$ is the wavelength of the incident X-ray beam, $2 \theta$ is the scattering angle and $n$ is an integer. Bragg's law demonstrates that the scattering pattern is the result of constructive interference of the scattered waves due to the interaction of X-rays to dominant structures of characteristic spacing $d$. The direct space $d$ is linked to $\theta$ and reciprocal space $q$ as shown in Eq. 2:

$q=\frac{4 \pi}{\lambda} \sin \theta=\frac{2 \pi}{d}$

During each SAXS measurement, the detector acquires a 2D intensity matrix. In this experiment, every SAXS measurement has an exposure time of 1 or 2 min depending on the test series. The intensity $I(q)$ is proportional to the number of scatterers contained in the illuminated volume. Therefore, highly repeated characteristic distances in the sample will result in scattered photons in specific scattering angles. Additionally, the orientation of a scatterer determines the azimuth of the scattered signal on the detector.

An example of a normalised SAXS pattern for the clay studied, corrected for the attenuation and scattering of the glass capillary by subtracting signal for an empty part of the capillary, is presented in Fig. 4. Structural information is extracted from radial sections averaged over an angle of $\pm 20^{\circ}$ of the 2D SAXS matrix, which produces $q$-value to intensity $I(q)$ plots (Fig. 5), whereas polar plots of intensity through azimuth at a fixed $q$-value are used to investigate

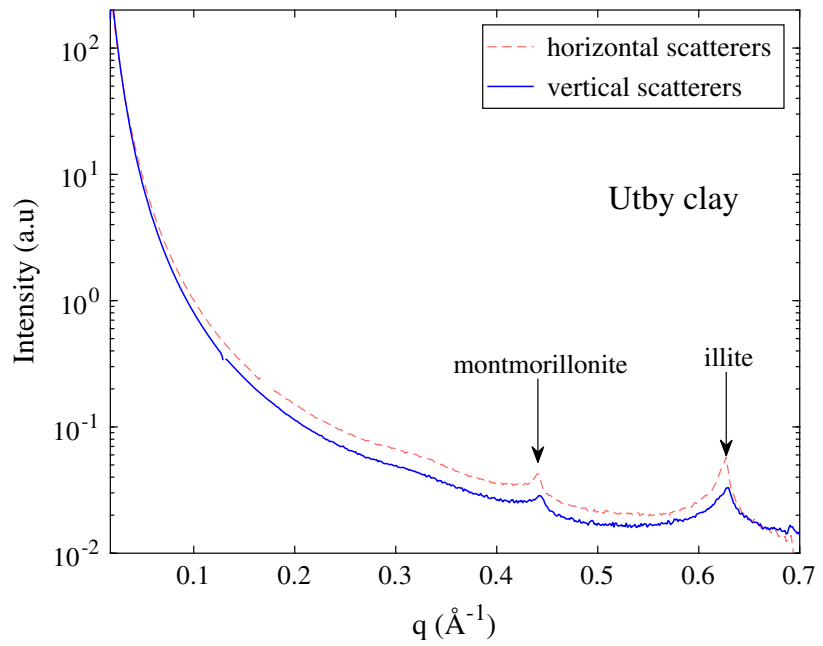

Fig. 5 1D curves of $q$-values to intensity $I(q)$ for Sample 1 . The red and blue coloured lines represent results for the corresponding sections in the detector image (Fig. 4). Characteristic features on the plot are the two distinct mineral peaks (colour figure online) 
the directional anisotropy of each mineral phase. Directional anisotropy information is yielded, since the absolute angle of orientation of a scatterer to the horizontal $\omega=90^{\circ}-\alpha$, where $\alpha$ is the azimuth of its scattered signal. The resolution limits of the technique, in terms of the structural length scales that can be assessed, are $D_{\max }$ and $D_{\min }[29]$.

$$
\begin{aligned}
& D_{\max }=\frac{\pi}{q_{\min }} \\
& D_{\min }=\frac{\pi}{q_{\max }}
\end{aligned}
$$

The largest distance that can be detected by the measurement configuration used is $D_{\max }=180 \AA$, calculated by Eq. 3. The lower limit $q_{\min }=0.0174 \AA^{-1}$ is governed by the collimator system. The smallest reliable distance is $D_{\min }=4.5 \AA$ (Eq. 4), which corresponds to $q_{\max }=0.7$ $\AA^{-1}$ and is controlled by the dimensions of the detector.

These resolution limits, coupled with the shape of the $I(q)$ curve at small $q$-values (no absolute maximum of the curve appears before the upper limit), limit the SAXS measurements in this test series to intra-particle spacings. Peaks in the SAXS curve reveal characteristic periodic distances in direct space, i.e. $d$-values. The evolution of $d$ values during mechanical loading can be monitored directly by shifts or broadening of the initial peaks that are, respectively, linked to uniform or non-uniform strain of the particles. An ellipse is fitted to the amplitudes of a given peak as a function of azimuth around the rings in the SAXS image, to extract additional information on the directional distributions of the scatterers. Prior to finding the peaks at each azimuth around a ring, the peaks were filtered by a Fourier filter (Fig. 6). Two parameters are selected to describe the variations with azimuth of the peaks: the index of directional anisotropy $(A)$ and the angle of principal orientation $(\phi) . A$ is defined as

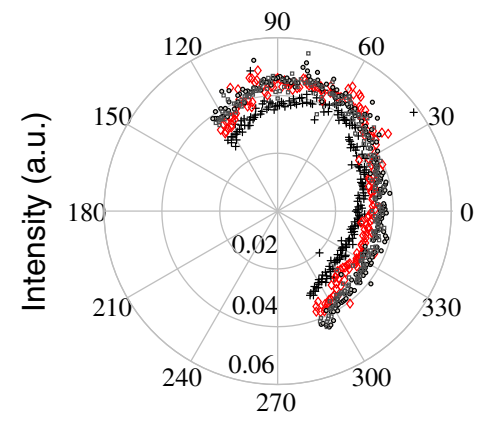

azimuth (degrees)

(a)

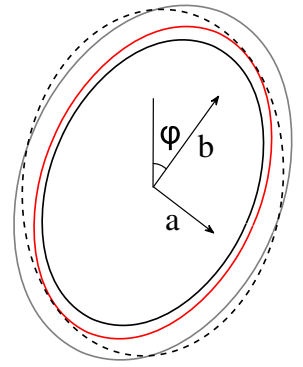

(b)
Fig. 6 Polar plot (a) of the normalised SAXS signal is parametrised by fitting an elliptical geometry (b)

$$
\begin{aligned}
A & =\frac{\text { major orientation intensity }}{\text { minor orientation intensity }}, \\
& =\frac{\text { semi-major axis of ellipse }(b)}{\text { semi-minor axis of ellipse }(a)}
\end{aligned}
$$

$A$ is not affected by the global rotation of the system and describes conditions from isotropic directional distribution (randomly oriented minerals) at values close to 1, to highly anisotropic states with $A \gg 1$ where the scatterers are strongly aligned to a certain direction. The second index $\phi$ (Fig. 6b) is the declination of the semi-major axis of the eclipse from the vertical, i.e. the angular difference of the largest directional fraction of planes from the horizontal plane. Thus, it expresses the global orientation of the system of scatterers in terms of an angle off horizontal.

\subsection{Materials tested}

The material studied is a natural sensitive silty clay from the Utby test site in Sweden. Its formal classification is high-plasticity clay, $\mathrm{CH}$, with high sensitivity $S_{t}$ [18]. The samples are characterised also by a high silt content ( $>20 \%$ by mass), typical for sensitive clays of the region. A summary of the geotechnical index properties is given in Table 1. In this material, the natural water content of the soil is significantly higher than the liquid limit of the reconstituted sample.

\subsection{Test plan}

The aim of this experimental series is to observe changes of structure at the nanometre scale when the material is probed mechanically towards new states of equilibrium. The details of the performed tests are summarised in Table 2 . Two types of stress paths are selected to trigger failure and phase transition of the sensitive clay.

\section{Analyses and results}

Two peaks are identified in the 1D intensity versus $q$ curve in Fig. 5. The two peaks correspond to the illite basal unit $(10 \AA)$ at $q=0.63 \AA^{-1}$ and montmorillonite mineral (14.28 $\AA$ ) at $q=0.44 \AA^{-1}$ [24]. Basal units are the layers that compose the clay particle. The evolution of peaks can monitor the intra-particle performance of the material. In addition to observations on the intra-particle spacing, it is possible to obtain information on the global directional anisotropy evolution of the material from the 2D SAXS data. A first sign of anisotropy is that the intensities of the scattering peaks are greater in the vertical direction, indicating that the number of horizontally aligned scatterers is 
Table 1 Soil characterisation with unit density $\rho$ plastic limit $w_{p}$; natural water content $w_{N}$, liquid limit $w_{l}$, plasticity index $P I$, sensitivity $S_{t}$ and undrained shear strength from fall cone test correlation $\tau_{f u}$

\begin{tabular}{llllllll}
\hline Depth $(\mathrm{m})$ & $\rho\left(\mathrm{t} / \mathrm{m}^{3}\right)$ & $w_{p}(\%)$ & $w_{N}(\%)$ & $w_{l}(\%)$ & $P I(\%)$ & $S_{t}(-)$ & $\tau_{f u}(\mathrm{kPa})$ \\
\hline 6 & 1.59 & 22 & 71 & 55 & 33 & 26 & 9 \\
\hline
\end{tabular}

Table 2 Summary table of performed tests

\begin{tabular}{llll}
\hline Sample & Description & Stages & Maximum stress (kPa) \\
\hline 1 & Monotonic compression & Loading, relaxation, unloading & 224 \\
2 & Cyclic loading & 3 Groups of cycles and a final monotonic part & 273 \\
\hline
\end{tabular}

systematically higher than the number of vertically aligned scatterers (Fig. 5).

\subsection{Monotonic compression test}

A compression test at high loading rate (i.e. high displaced volume rate) was performed on Sample 1 (Fig. 7). The onesided drainage boundary of the specimen and the high loading rate used reproduce partially drained conditions through the specimen during monotonic loading stages. The stress-strain curve is used to characterise the mechanical response of the sample to the external loading. A local correction of strains is obtained around the point of

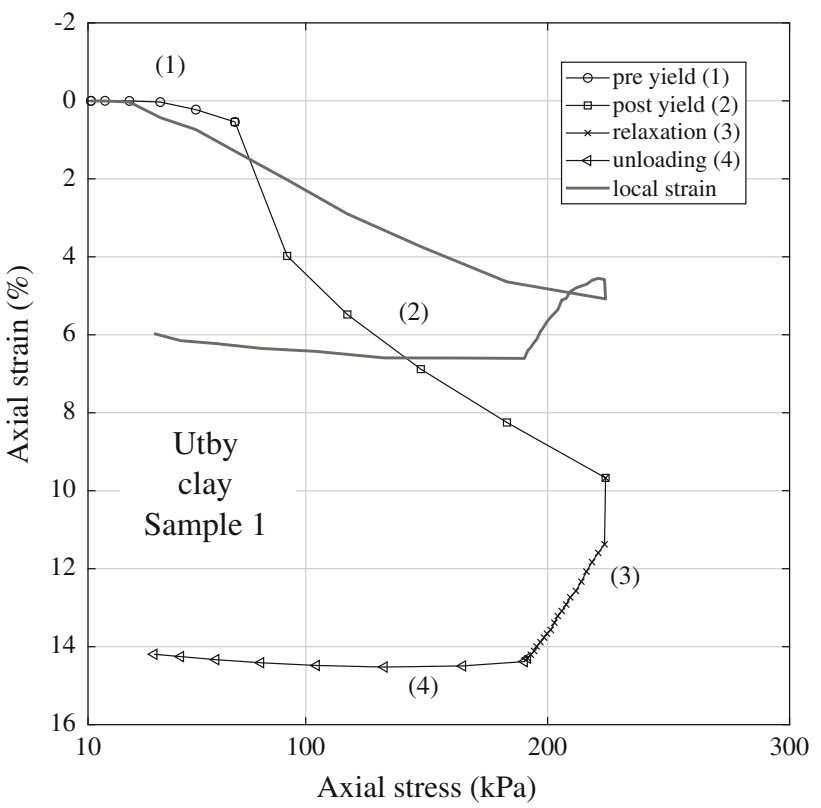

Fig. 7 High loading rate monotonic compression $(17.4 \mathrm{kPa} / \mathrm{min})$, representing undrained loading conditions. Strain is calculated by digital image correlation analysis both for total length reduction in the specimen i.e. global strain (1-4), as well as for a small reference area around the X-ray illuminated area, i.e. local strain
SAXS measurement using the DIC data, which is plotted against the externally applied stress for comparison with the sample response, but this curve does not represent the local stress-strain behaviour. The complete stress path for this test consists of four parts: pre-yield, post-yield, relaxation and unloading. In the context of this paper, the interpretation of yield refers to the transition of the material response from largely recoverable strains to largely irrecoverably (permanent) strains. During the first two loading stages, the loading rate was constant at $17.4 \mathrm{kPa} /$ min. In the next 'relaxation' stage, no additional energy was added to the system (pump halted). The sample, however, continued to deform due to consolidation (flow of pore water). In the final stage, the sample was unloaded. The four stages are labelled as distinct sections of the total stress-strain curve (Fig. 7). The stress values refer to axial total stresses applied at the top surface of the specimen. It was not possible to reliably obtain effective stress as its physical interpretation cannot be applied at the clay particle level where electrical and capillary forces dominate. Simultaneous SAXS measurements and digital images were acquired with a 60-s interval. The SAXS $q-I(q)$ curves for each loading point (Fig. 7) are plotted in Figs. 8 and 9 for the vertical and $30^{\circ}$ off-horizontal spacing, respectively. Horizontal spacing was not considered for plotting due to discontinuities in the detector panel close to the $q$-values of interest for one of the minerals. No significant change in the SAXS data (i.e. position and shape of the peaks) can be observed during the first two monotonic parts of the loading path, except for a uniform shift of the intensity curve upwards. This increase in intensity is to be expected, as intensity values indicate the amount of scatterers inside the illuminated volume. So, post-yield, i.e. for higher strain rates a larger number of scatterers pass through the reference volume during exposure time and the material also consolidate. A clear change in the scattering signal is the appearance of a new peak at larger spacing for 


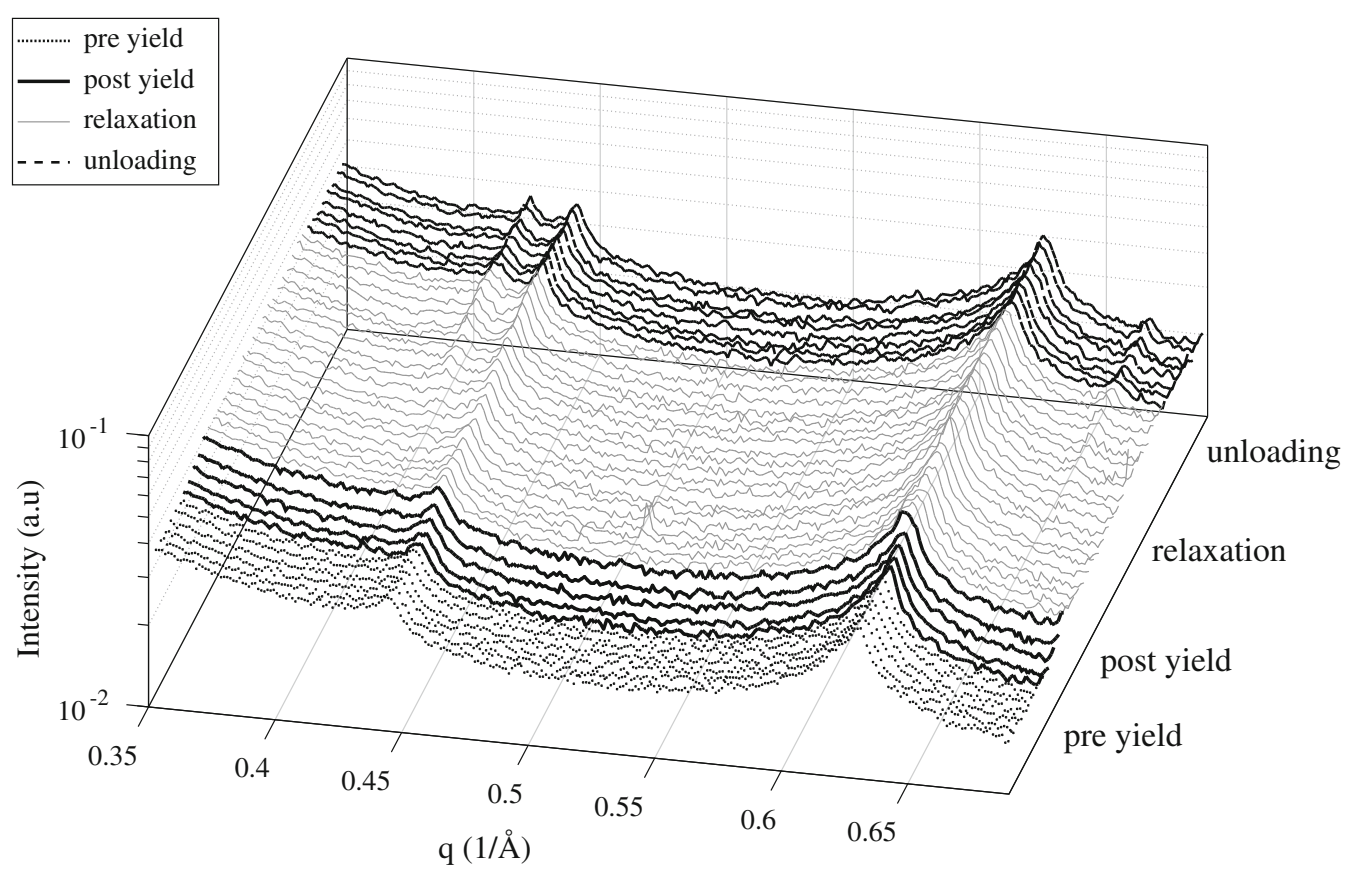

Fig. $83 \mathrm{D}$ plot of $q$-range to intensity curves of the vertically aligned scattering planes. A new peak appears during the relaxation stage

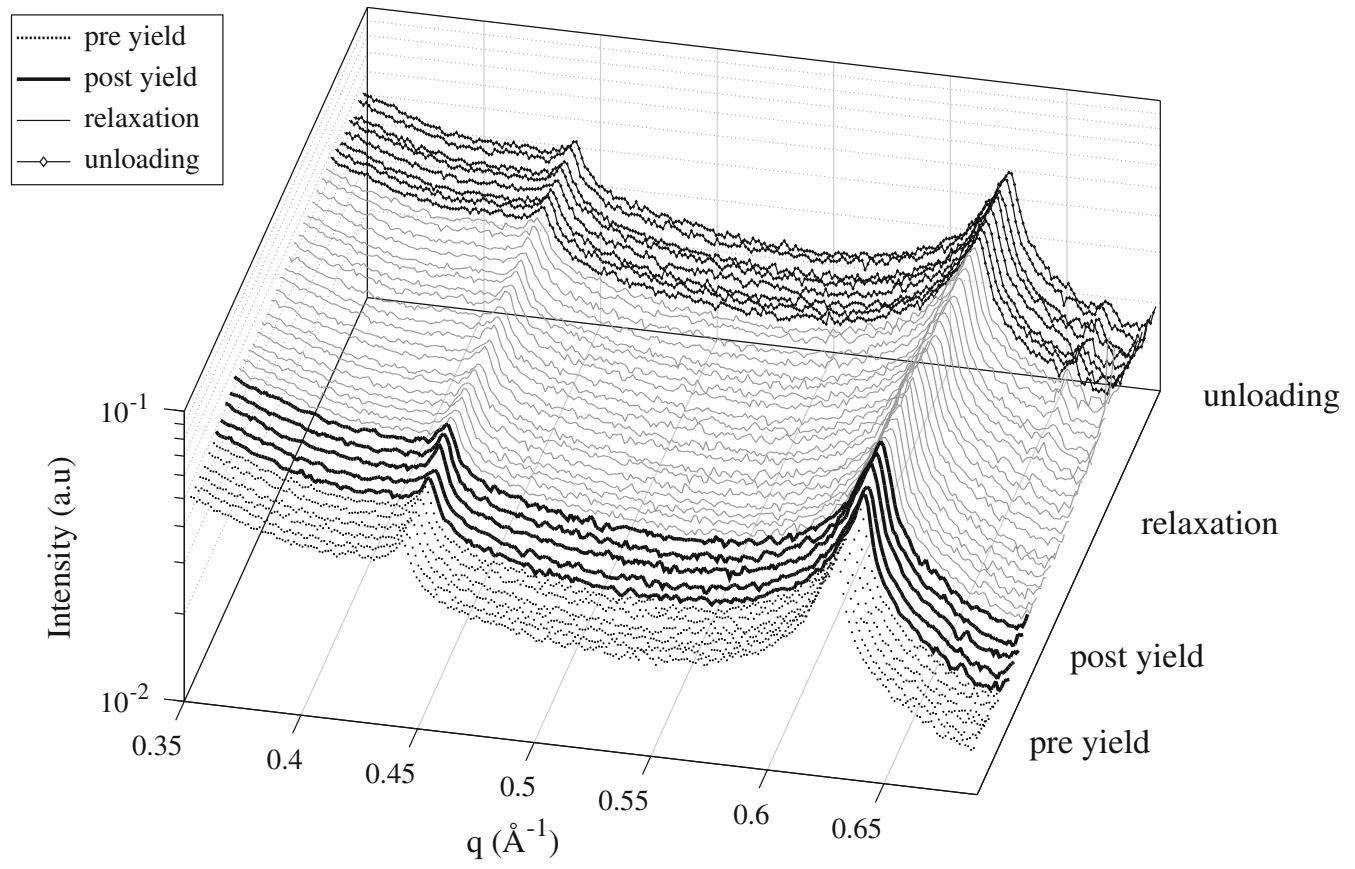

Fig. $93 \mathrm{D}$ plot of $q$-range to intensity curves of the aligned $30^{\circ}$ of horizontal scattering planes

the expandable mineral during the relaxation and unloading stages. The new peak is distinguishable in the vertical spacing (Fig. 8). Nevertheless, the disproportionate increase in intensity between the minerals, which indicates the existence of new spacing for montmorillonite, is seen for the entire azimuth range as shown in Fig. 10).

\subsection{Cyclic test}

Another loading path and its influence on the structure that is of great interest is load reversal. As shown in Table 3, groups of loading cycles with gradually increasing average amplitude (stages 1-3) are applied to Sample 2. In the final stage, 4 , the sample was compressed further to high stress levels. SAXS measurements were conducted after each 


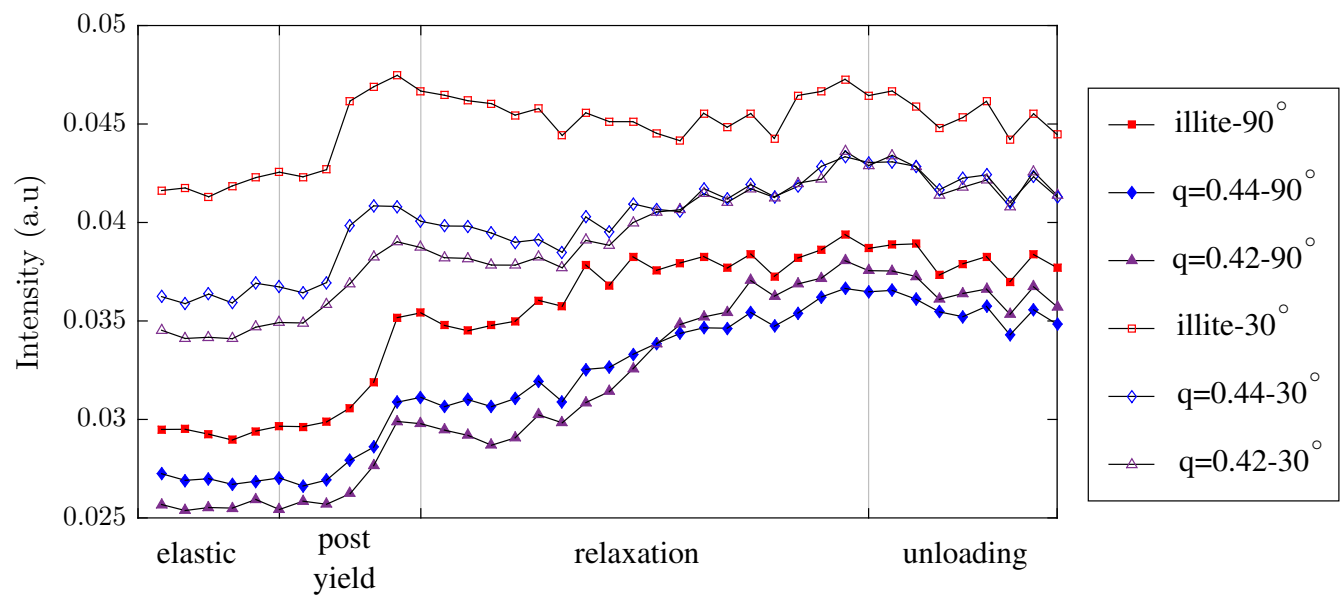

Fig. 10 Evolution of intensity of reciprocal spacing of illite and of the two discrete reciprocal spacing of montmorillonite through different loading steps, for vertically aligned planes and $30^{\circ}$ off horizontal. Relative increase in intensity for the new peak $\left(q=0.42 \AA^{-1}\right)$ is observed. Intensity values for each peak are products of averaging for a 10-pixel wide zone around the centre of the peak

Table 3 Loading scheme for cyclic stress path of Sample 2

\begin{tabular}{|c|c|c|c|c|}
\hline Stage & Number of cycles & Accumulative number of cycles & Average amplitude $(\mathrm{kPa})$ & Standard deviation $(\mathrm{kPa})$ \\
\hline \multicolumn{5}{|c|}{ Cyclic loading } \\
\hline 1 & 5 & 5 & 43.0 & 12.9 \\
\hline 2 & 10 & 15 & 59.1 & 12.1 \\
\hline 3 & 5 & 20 & 96.4 & 7.7 \\
\hline Stage & & Stress rate $(\mathrm{kPa} / \mathrm{min})$ & & Maximum value $(\mathrm{kPa})$ \\
\hline \multicolumn{5}{|c|}{ Monotonic loading } \\
\hline 4 & & 20.7 & & 272.5 \\
\hline
\end{tabular}

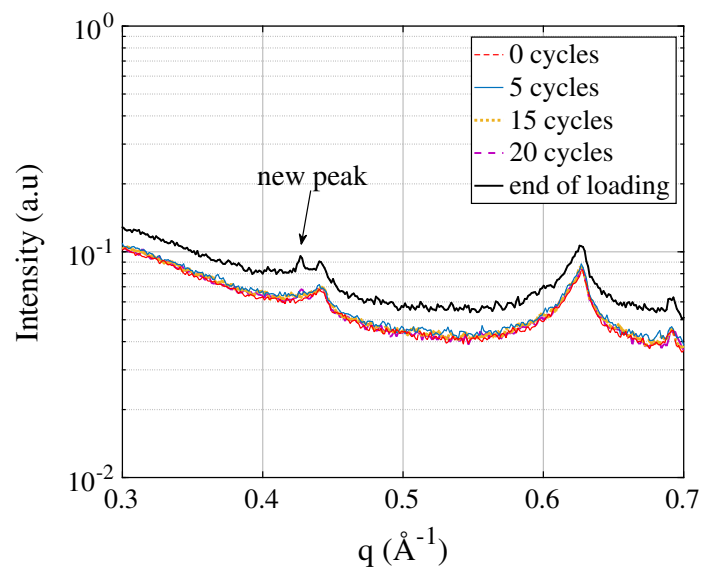

Fig. 11 SAXS 1D curve plots of the $30^{\circ}$ of horizontal scattering planes after different loading groups of the cyclic test

loading group as well after the end of loading with an exposure time of $120 \mathrm{~s}$ for each SAXS acquisition.

The SAXS intensity plots (Figs. 11, 12) indicate no discernible effect when the cyclic loading groups are

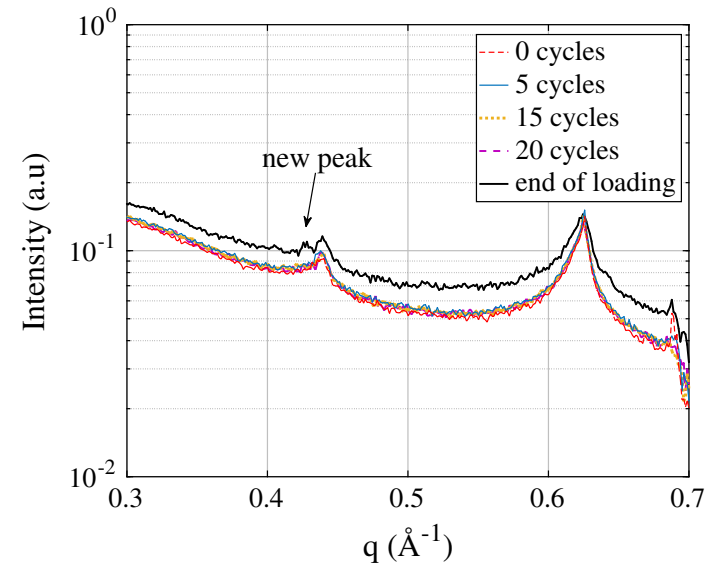

Fig. 12 SAXS 1D curve plots of the vertical scattering planes after different loading groups of the cyclic test

imposed. Furthermore, after the end of the test and fast final loading, again the additional SAXS peak appears around the same new position as observed in the case of Sample 1, next to the peak of the secondary mineral. 


\subsection{Physical interpretation}

A key observation of the tests was the appearance of a new peak after the loading, which is an indication of a new scattering length (i.e. structural scale) in the material, rather than any indication of nanoscale strain, which would give rise to a shift or broadening of existing peaks (for uniform or non-uniform strain, respectively). Moreover, an expected mechanism of strain of an expandable mineral is absorption of a water layer between the basal units. This is not the case here, as the increase in spacing is only $0.57 \AA$, significantly smaller then the Van der Waals diameter of water molecules which is around $3.25 \AA$ [19].

The new peak is not an artefact of the test cell, as tests on intact and disturbed extruded material from the capillary placed between two kapton films (highly X-ray transparent material) corroborate the results (Fig. 13). A new broad peak centred at $q=0.40 \AA^{-1}(15.76 \AA)$ again appears for larger basal spacing, supporting the results of the reported compression tests.

An important aspect is that the new scale length appears only after the 'undrained' mechanical perturbation. In finegrained soils, the principal mechanism governing the deformation (decomposed in deviatoric and volumetric strain) triggered by the increase in total stress is the delayed increase in effective stress resulting from the dissipation of excess pore water pressures. The hydraulic properties of the pore network and drainage conditions dictate the characteristic time scale associated with this pore water flow mechanism. This influences primarily the pore system structure, but should not effect directly intra-particle-level structure as can be seen in the current measurements. Coupled with the thixotropic behaviour of the material, the new scale length perhaps should be explained through other intrinsic mechanisms at the particulate scale.

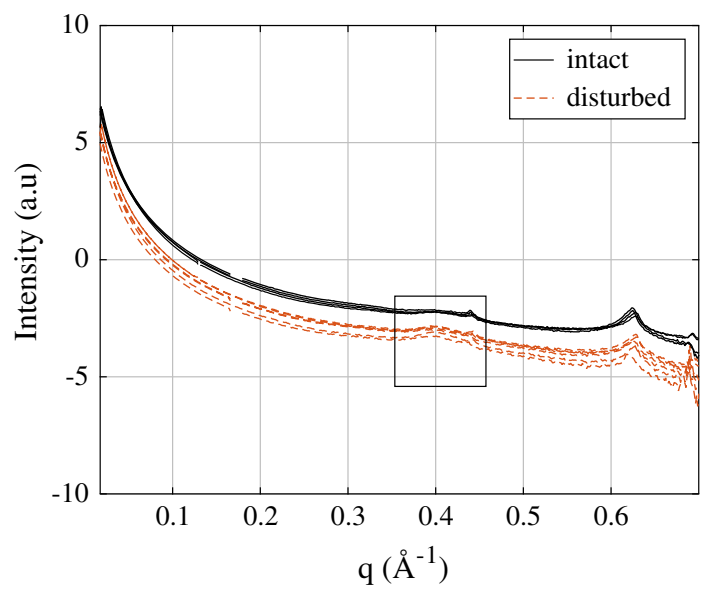

Fig. 13 Group of measurements for intact and mechanically disturbed samples
One of the governing mechanisms for the colloidal behaviour of clays is isomorphous substitution, i.e. the exchange of cations of similar size and valence whilst the crystal structure is preserved. This process is completed during the formation of minerals and is the factor that determines the permanent charge of the mineral and thus its cation exchange capacity. Combined with the pore water chemistry, this permanent charge of the clay mineral governs the behaviour of the colloid system. Isomorphous substitution is significant in expandable minerals. Smectite minerals have high cation exchange capacities (CEC), in the range of $80-150 \mathrm{meq} / 100 \mathrm{~g} \mathrm{[24]}$ and are vulnerable to charge changes. Therefore, the emerging scale length in the SAXS data could be attributed to replacement of cations, especially from the interlayer exchangeable cations due to the new charge equilibrium of the global clay stacked structure. Large pore water gradients, and hence flow rates, present in the monotonic loading stages would not allow a stable ionic composition around the particles, required for an equilibrium state. On the other hand, illite, which is a low cation exchange capacity mineral, does not show similar behaviour. Nonetheless, average increase in spacing of values around $1 \AA$ is relatively small, close to the bond length of covalent bonds.

As opposed to montmorillonite, illite maintains a stable structure during and after loading. Although the two minerals show different basal unit response, they seem to have similar global directional evolution as can be seen by the evolution of the directional parameters $A$ and $\phi$ (Figs. 14, 15). The directional anisotropy as described by index $A$ decreases after the mechanical perturbation for both minerals (Figs. 14b, 15b). Additionally, the evolution of principal orientation $\phi$ indicates that whilst the same trends emerge for the two minerals, their absolute orientation distributions differ (Figs. 14a, 15a). These observations, further supported by the peak asymmetry of illite, are indications of a mixed turbostratic mineral system. Turbostratic disorder is described as a crystal structure in which basal planes have slipped out of alignment [22]. Such disorder is observed in layered materials, such as clay minerals and graphite structures. In clay minerals, turbostratic disorder is more common for montmorillonite stacks, but for the current case peak asymmetry is observed for the illite basal unit spacing only. Also, turbostratic formations are often linked to interstratified clays composed of illite-smectite mixed particles.

\section{Discussion and conclusions}

The presented new experimental method combines a miniature plane-strain compression setup to test undisturbed wet clay samples with simultaneous nanoscale 


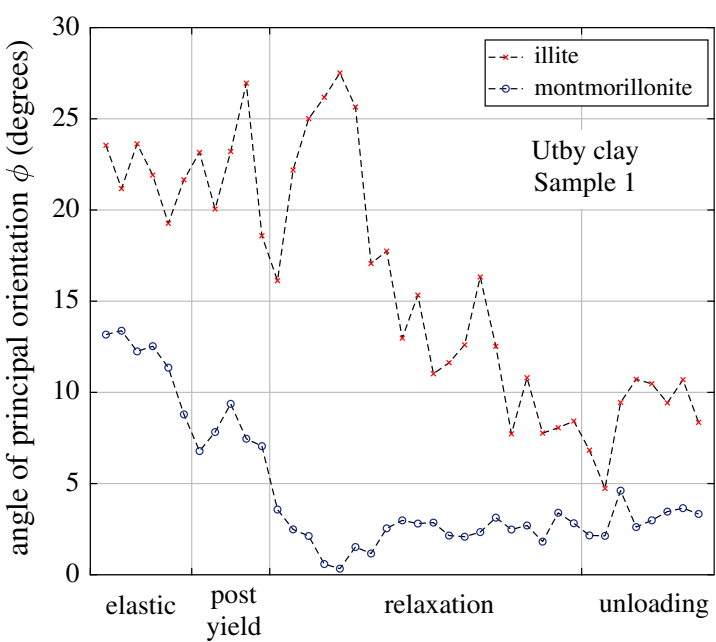

(a)

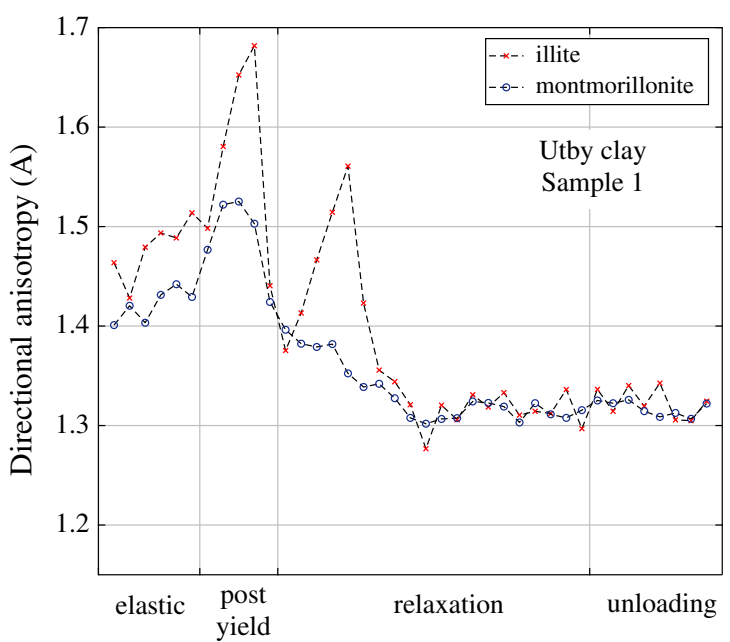

(b)

Fig. 14 Angle of principal orientation $\phi$ (a) and directional anisotropy $(A)$ index (b) evolution during loading stages for Sample 1

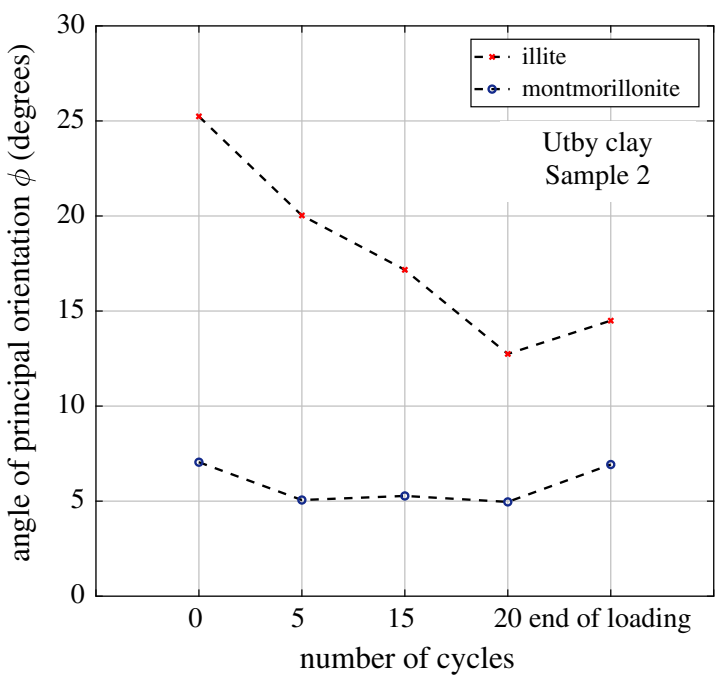

(a)

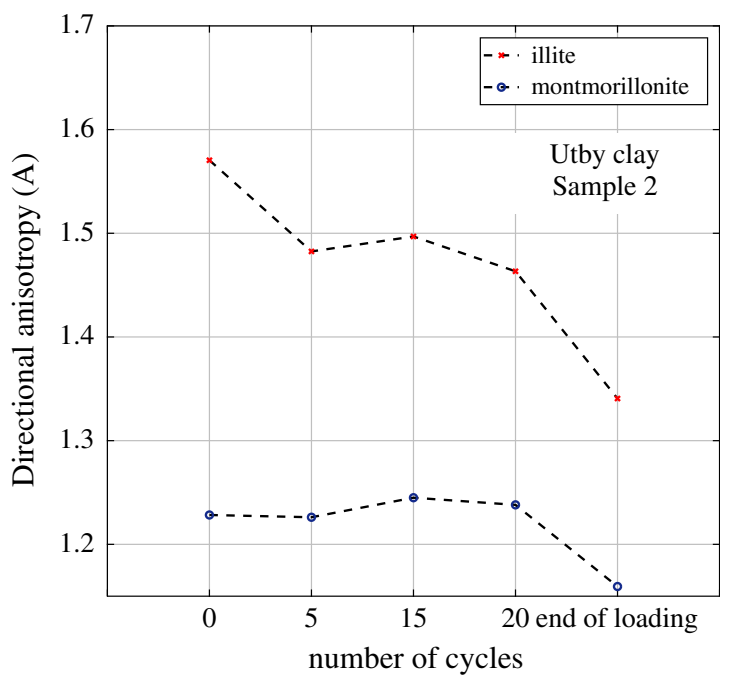

(b)

Fig. 15 Angle of principal orientation $\phi$ (a) and directional anisotropy $A$ index (b) evolution during loading stages for Sample 2

observations using Small Angle X-ray Scattering and macro-scale Digital Image Correlation for extraction of strain. The feasibility of the test setup is demonstrated for a natural sensitive clay from Sweden. Nanoscale observations on the evolving intra-particle response of clay during hydro-mechanical loading are presented in addition to assessing its composition.

A primary outcome of the work is that no significant structural change was observed at intra-particle level during mechanical loading for the clay investigated. At rest stages, during relaxation or after loading, new peaks appear in the SAXS data. These peaks indicate a new structural scale for the expandable mineral. This response is consistent with the thixotropic macroscopic behaviour of sensitive clays, linking rearrangement of micro-structure components to the rheological performance of the material. The main difference between these two states is the magnitude of flow gradient. An interpretation would be that, at rest states, lower water flow gradients in the pore system can allow clay particles to establish a new electrochemical equilibrium through ion exchange.

$\mathrm{X}$-ray scattering shows great promise as a method to study (sensitive) clays in their undisturbed state providing unique insight using bulk measurements. As opposed to other microscopy techniques, sample preparation is relatively benign. Moreover, fast acquisition rates (maximum two minutes in this particular set up) are beneficial to realtime study of time-dependent mechanisms; faster rates are possible with more intense sources. It is demonstrated that with little additional effort, the method can be 
complemented with classic hydro-mechanical testing of the material. As an extension of the presented work, it would be worthwhile to broaden the observation scale to smaller and larger spacings using Wide Angle X-ray Scattering (WAXS) and Ultra Small Angle X-ray Scattering (USAXS) to cover the complete spatial spectrum of clay structure components complementary to the inter-particle scale.

Acknowledgements The funding from the Swedish Research Council (Vetenskapsrådet) under Grant No. 491401 is greatly acknowledged. Further financial support was received for performing the experiments at the I911-4 beam line at MaxIV under Experiment Nos. 20140129 and 20140497. Additionally, SL acknowledges the Federal Ministry of Education and Research (BMBF) for the financial support via the SAXS/SANS Project (05K16WE1).

Open Access This article is distributed under the terms of the Creative Commons Attribution 4.0 International License (http://creative commons.org/licenses/by/4.0/), which permits unrestricted use, distribution, and reproduction in any medium, provided you give appropriate credit to the original author(s) and the source, provide a link to the Creative Commons license, and indicate if changes were made.

\section{References}

1. Barnes HA (1997) Thixotropy-a review. J NonNewton Fluid Mech 70:1-33

2. Bohor BF, Hughes RE (1971) Scanning electron microscopy of clay and clay minerals. Clays Clay Miner 19:49-54

3. Bornert M, Valés F, Gharbi H, Minh DN (2010) Multiscale fullfield strain measurements for micromechanical investigations of the hydromechanical behaviour of clayey rocks. Strain 46:33-46

4. Brochard L, Honório T, Vandamme M, Bornert M, Peigney M (2017) Nanoscale origin of the thermo-mechanical behavior of clays. Acta Geotech 12:1261-1279

5. Casagrande A (1932) The structure of clay and its importance in foundation engineering. Boston Soc Civil Eng J 19:168-209

6. Chassagne C, Mietta F, Winterwerp JC (2009) Electrokinetic study of kaolinite suspensions. J Colloid Interface Sci 336:352-359

7. Correlated Solutions, Vic-3d 2010, reference manual

8. Cotecchia F, Chandler RJ (1997) The influence of structure on the pre-failure behaviour of natural clay. Géotechnique 47:523-544

9. Deirieh A (2016) From clay slurries to mudrocks: a cryo-SEM investigation of the development of the porosity and microstructure, Ph.D. thesis, Massachussets Institute of Technology

10. Deirieh A, Chang IY, Whittaker ML, Weigand S, Keane D, Rix J, Germaine J, Joester D, Flemings PB (2018) Particle arrangements in clay slurries: the case against the honeycomb structure. Appl Clay Sci 152:166-172

11. Delage P, Lefebvre G (1984) Study of the structure of a sensitive champlain clay and of its evolution during consolidation. Can Geotech J 21:21-35

12. Ebrahimi D, Pellenq RJ-M, Whittle AJ (2012) Nanoscale elastic properties of montmorillonite upon water adsorption. Langmuir 28:16855-16863

13. Engqvist J, Hall SA, Wallin M, Ristinmaa M, Plivelic TS (2014) Multi-scale measurement of (amorphous) polymer deformation: simultaneous X-ray scattering, digital image correlation and insitu loading. Exp Mech 54:1373-1383

14. Guinier A, Fournet G (1955) Small-angle scattering of X-rays. Wiley, New York

15. Hattab M, Fleureau J-M (2010) Experimental study of kaolin particle orientation mechanism. Géotechnique 60:323-331

16. Hattab M, Hammad T, Fleureau J-M, Hicher P-Y (2013) Behaviour of a sensitive marine sediment: microstructural investigation. Géotechnique 63:71-84

17. Hicher PY, Wahyudi H, Tessier D (2000) Microstructural analysis of inherent and induced anisotropy in clay. Mech Cohesive Frict Mater 5:341-371

18. Karlsson M, Emdal A, Dijkstra J (2016) Consequences of sample disturbance when predicting long-term settlements in soft clay. Can Geotech J 53:1-13

19. Katti DR, Srinivasamurthy L, Katti KS (2015) Molecular modeling of initiation of interlayer swelling in Na-montmorillonite expansive clay. Can Geotech J 52:1385-1395

20. Labrador A, Cerenius Y, Svensson C, Theodor K, Plivelic T (2013) The yellow mini-hutch for SAXS experiments at MAX IV Laboratory. J Phys Conf Ser 425:072019

21. Lambe TW, Whitman RV (1969) Soil mechanics. Wiley, New York

22. Lutterotti L, Voltolini M, Wenk HR, Bandyopadhyay K, Vanorio $\mathrm{T}$ (2010) Texture analysis of a turbostratically disordered Camontmorillonite. Am Mineral 95:98-103

23. Matusewicz M, Liljeström V, Muurinen A, Serimaa R (2011) SAXS and TEM investigation of bentonite structure. In: NEA CLAY CLUB workshop proceedings. Karlsruhe, Germany

24. Mitchell JK, Soga K (2005) Fundamentals of soil behavior, 3rd edn. Wiley, New York

25. Olphen HV (1964) Internal mutual flocculation in clay suspensions. J Colloid Sci 19:313-322

26. Paineau E, Dozova I, Philippe A, Bihannic I, Meneau F, Baravian C, Michot LJ, Davidson P (2012) In-situ SAXS study of aqueous clay suspensions submitted to alternating current electric fields. J Phys Chem B 116:13516-13524

27. Petukhov AV, Meijer J, Vroege GJ (2015) Particle shape effects in colloidal crystals and colloidal liquid crystals: small-angle $\mathrm{X}$-ray scattering studies with microradian resolution. Curr Opin Colloid Interface Sci 20:272-281

28. Santamarina JC (2003) Soil behavior at the microscale: particle forces. In: Proceedings of a symposium on soil behavior and soft ground construction in honor of Charles C. Ladd, MIT, Oct 2001

29. Schnablegger H, Singh Y (2013) The SAXS guide: getting acquainted with the principles. Anton Paar, Graz

30. Simakumar V, Doran IG, Graham J (2002) Particle orientation and its influence on the mechanical behaviour of isotropically consolidated reconstituted clay. Eng Geol 66:197-209

31. Torrance JK (1983) Towards a general model of quick clay development. Sedimentology 30:547-555

32. Wang Y-H, Siu W-K (2006) Structure characteristics and mechanical properties of kaolinite soils. I. Surface charges and structural characterizations. Can Geotech J 43:587-600

33. Yang DS, Bornert M, Chanchole S, Valli P, Gatmiri B (2011) Experimental investigation of the delayed behaviour of unsaturated argillaceous rocks by means of digital image correlation techniques. Appl Clay Sci 54:53-62

34. Zhang L, Jahns C, Hsiao BS, Chu B (2003) Synchrotron SAXS/ WAXD and rheological studies of clay suspensions in silicone fluid. J Colloid Interface Sci 266:339-345

Publisher's Note Springer Nature remains neutral with regard to jurisdictional claims in published maps and institutional affiliations. 